\section{Kondom-assoziierte Erektionsstörung steht oft nicht allein}

\begin{abstract}
Dem Versuch, ein Kondom zu benutzen, kommt bisweilen das Zielobjekt abhanden: Erektionsstörungen im Zusammenhang mit dem Kondomgebrauch treten auch bei jüngeren Männern auf - mit Tendenz zur Generalisierung.
\end{abstract}

$E^{\text {in }}$ in Team des Kinsey Institute for Research in Sex, Gender, and Reproduction, Bloomington/USA, um Stephanie Sanders hat für eine Studie zu Erektionsstörungen von Kondomnutzern 479 junge heterosexuelle Männer zwischen 18 und 24 Jahren untersucht. Ziel war es herauszufinden, ob Kondom-assoziierte Erektionsschwächen (KAES) auf allgemeinere Erektionsprobleme hinweisen.

38,4 \% der Männer wurden als NichtKAES klassifiziert, 13,8 \% berichteten von zumindest gelegentlichem Erektionsverlust während des Anlegens eines Kondoms, 15,7 \% verloren während des Verkehrs ihre Erektion. 32,2 \% ereilte das Missgeschick schon in beiden Situationen. Der abgefragte Zeitraum erstreckte sich jeweils auf die zurückliegenden drei Monate. Auch wenn dies keinem häufig geschah, berichteten Männer mit KAES öfter über allgemeine, Kondom-unabhängige Erektionsprobleme als Männer ohne KAES. Über ein gelegentliches Erschlaffen vor der Penetration ohne Kondom berichteten 9,9\% der Letzteren. In den KAES-Gruppen waren es zwischen 23,6 und $43,0 \%$. Nach dem International Index of Erectile Function (IIEF-5) war bei 8,7 \% der Männer ohne KAES eine Erektionsstörung (ED) festzustellen. In der Gruppe, die nur Probleme beim Anlegen des Kondoms gehabt hatte, waren es $18,2 \%$. Im Fall von Verkehrsstörungen mit Kondom lag die ED-Quote bei 22,7\%, in der kombinierten Gruppe erreichte sie 31,8 \%.
Allerdings handelte es sich überwiegend um leichte Störungen, eine schwere ED war bei keinem der Männer festzustellen.

Fazit: Sanders und Kollegen erklären sich die Phänomene damit, dass Männer mit generellen Erektionsproblemen auch beim Gebrauch von Kondomen mit diesem Schwierigkeiten zu kämpfen haben dürften. Sie halten es aber auch für denkbar, dass Männer, die beim Benutzen von Kondomen situationsbedingt einen Misserfolg erlebt haben, sich um ihre Standfestigkeit sorgen - und anfälliger für eine weiter ausgreifende ED werden. Dies sei zwar spekulativ, so Sanders et al. „Es stünde aber durchaus im Einklang mit anderen Erkenntnissen, wonach kognitive und emotionale Faktoren wie Beunruhigung und Ablenkung einen wichtigen Part darin spielen, eine $\mathrm{ED}$ entstehen zu lassen und aufrechtzuerhalten." Dr. Robert Bublak

Sanders SA et al. General erectile functioning among young, heterosexual men who do and do not report condom-associated erection problems (CAEP). J Sex Med. 2015 doi: 10.1111/ jsm.12964.

\title{
Medikamente helfen nicht beim spontanen Steinabgang
}

\section{Weder Tamsulosin noch Nifedipin förderten einer aktuellen britischen Studie zufolge den Spontanabgang von Harnleitersteinen. Beide bewirken nicht mehr als ein Placebo.}

$D^{i}$ ie Wahrscheinlichkeit, dass ein Harnleiterstein spontan abgeht, hängt von Größe und Lage ab. Sofern keine Komplikation wie etwa ein Harnstau vorliegt, ist eine konservative Therapie meist ausreichend. Hilfreich wären Medikamente, die den Spontanabgang unterstützen. In der deutschen wie in anderen Leitlinien zur Urolithiasis werden für eine solche medikamentöse expulsive Therapie (MET) besonders der Alphablocker Tamsulosin und der Kalziumantagonist Nifedipin empfohlen. Sie sollen eine Relaxation der glatten Muskulatur bewirken. In Metaanalysen fanden sich Hinweise, dass beide diesen Zweck erfüllen. Allerdings zeigten die Analysen auch die mangelnde Güte der vorhandenen Daten und die
Notwendigkeit von qualitativ hochwertige Studien. Diese Lücke haben Robert Pickard von der Newcastle University und seine Kollegen mit der multizentrischen, randomisierten placebokontrollierten Studie „Spontaneous Urinary Stone Passage Enabled by Drugs" (SUSPEND) zu schließen versucht:1.167 Patienten mit symptomatischen Uretersteinen $(\leq 10 \mathrm{~mm})$ wurden drei Gruppen zugeordnet. Probanden der ersten Gruppe bekamen vier Wochen lang täglich 400 $\mu \mathrm{g}$ Tamsulosin verordnet, die Patienten der zweiten Gruppe $30 \mathrm{mg}$ Nifedipin, die dritte Gruppe Placebo. Primärer Endpunkt der Studie war der Anteil der Patienten in den jeweiligen Studienarmen, die innerhalb von vier Wochen nach der
Randomisierung keiner weiteren Intervention mehr zur Befreiung von Konkrementen bedurften.

Gleichgültig, ob die Patienten eine Wirksubstanz oder Placebo eingenommen hatten - ein Unterschied in der Wirkung war nicht festzustellen. Keiner weiteren Eingriffe bedurften $81 \%$ der Probanden unter Tamsulosin, $80 \%$ unter Nifedipin und $80 \%$ in der Placebogruppe. Zwischen aktiver Therapie und Placebo bestand ebenso wenig eine statistisch relevante Differenz $(\mathrm{p}=0,78)$ wie zwischen Tamsulosin und Nifedipin $(\mathrm{p}=0,77)$.

Fazit: Jean de la Rosette und Pilar Laguna von der Urologischen Klinik der Universität Amsterdam kommentierten in ihrem Befund zur Studie, dass die Ergebnisse die positiven Erwartungen an die Gabe von Alphablockern zur Therapie von Uretersteinen nicht bestätigen würden.

Dr. Robert Bublak

Pickard $R$ et al. Medical expulsive therapy in adults with ureteric colic: a multicentre, randomised, placebo-controlled trial. Lancet. 2015; doi: 10.1016/S0140-6736(15)60933-3. 